(C) [2010] IEEE. Reprinted, with permission, from [Dr Zenon Chaczko, Christopher Chiu, Anup Kale, Cooperative Agent-based SANET Architecture for Personalised Healthcare Monitor, Signal Processing and Communication Systems (ICSPCS), 2010 4th International Conference on 13-15 Dec. 2010]. This material is posted here with permission of the IEEE. Such permission of the IEEE does not in any way imply IEEE endorsement of any of the University of Technology, Sydney's products or services. Internal or personal use of this material is permitted.

However, permission to reprint/republish this material for advertising or promotional purposes or for creating new collective works for resale or redistribution must be obtained from the IEEE by writing to pubs-permissions@ieee.org. By choosing to view this document, you agree to all provisions of the copyright laws protecting it 


\title{
Cooperative Agent-based SANET Architecture for Personalised Healthcare Monitoring
}

\author{
Dr Zenon Chaczko ${ }^{1}$, Christopher Chiu ${ }^{2}$, Anup Kale ${ }^{3}$
}

\begin{abstract}
The application of an software agent-based computational technique that implements Extended Kohonen Maps (EKMs) for the management of Sensor-Actuator networks (SANETs) in health-care facilities. The agent-based model incorporates the BDI (Belief-Desire-Intention) Agent paradigms by Georgeff et al. EKMs perform the quantitative analysis of an algorithmic artificial neural network process by using an indirect-mapping EKM to self-organize. Current results show a combinatorial approach to optimization with EKMs provides an improvement in event trajectory estimation compared to standalone cooperative EKM processes to allow responsive event detection for patient monitoring scenarios. This will allow healthcare professionals to focus less on administrative tasks, and more on improving patient needs, particularly with people who are in need for dedicated care and round-the-clock monitoring.
\end{abstract}

Keywords - BDI Agent Framework, Extended Kohonen Maps (EKM), Healthcare Infrastructures, Sensor Actuator Networks (SANETs)

\section{PURPOSE OF SANET INFRASTRUCTURES}

A distributed SANET infrastructure using biomimetic principles for active monitoring of patients in healthcare is an on-going concern for modern medical practice. Current health practice is overly dependent upon existing passive communication devices, such as pagers and buzzers that can potentially distract staff from their daily tasks. Furthermore, the priority of these notices must be made by the staff alone, as these systems do not have the capability to measure which patient requires the most attention, so there is a greater responsibility to ensure staff have ease of access to patient record data at a moment's notice. For these main reasons, an evolvable network that encapsulates monitoring and patient record processes for health care will augment existing practices by tracking the wellbeing of a patient into their recovery.

The design of a SANET-based infrastructure for healthcare needs to adhere to industry standards including HL7 [10], but also take into consideration the human-user interaction needs for the end user. From a technical standpoint, efficient and concise data exchange is an imperative need for actively tracking and monitoring changes in event, but also to ensure that a systemic infrastructure suitable for SANET environments [6][7] is compatible with health care professional needs for ensuring the well-being of their patients in care and rehabilitation.

\footnotetext{
i,2,3 Dr Zenon Chaczko, Chris Chiu and Anup Kale are with the Faculty of Engineering \& IT, University of Technology, Sydney, Australia (E-mail: \{zenon.chaczko, christopher.chiu, anup.v.kale\}@uts.edu.au)
}

From a qualitative standpoint, the SANET infrastructure model must be designed with hard real-time criticality and fault-tolerance, while incorporating redundancy to ensure the data responses and feedback is delivered within finite limits. Thus, the infrastructure must achieve strict Quality of Service (QoS) qualitative requirements [3][21], inclusive of scalability, robustness, security, privacy and efficiency. The SANET infrastructure should support the process of software development by facilitating integration of components and reducing the accidental complexities related to heterogeneous environments in health institutions. The Cooperative-Oriented Sensors Actuators model (COSA) by Chaczko [5] is used to represent a SANET neighborhood and its moderation by two global aspects: the accuracy of a node's position and the node's immunity to error propagation.

The algorithmic categories that are considered in the experimental model are ordered from local to global domain concerns. These include the following concepts of feature mapping of sensory inputs (using neural network techniques), multi-variate regression to reduce sampling errors, and multi-objective heuristics to provide future learning capability into the system:

- $\quad$ Feature Mapping:

Utilizing an Extended Kohonen Map (EKM) by Kohonen [17][18], the self-organizing map partitions sensory-aware spaces discretely; thus the generalization capability arises from its selforganization during map training [19]. Map resolution is improved in frequently encountered stimuli regions, thus mimicking biological sensory perceptions where reinforced practice allows for prediction of anticipated events.

- Multi-variate Regression Techniques:

Formulated as a non-linear multi-variate regression problem [11][13][14], the main issue is that training samples must be regularly collected for error sampling rates. The sampling process is simplified by providing qualitative feedback at the end of the executing control sequence [14]. This technique ensures smoothness when local minima or maxima results are encountered, such that sensor variations are minimized.

- Multi-Objective Meta-Heuristics:

Reinforcement learning heuristics, based upon nondeterministic biological concepts, provides a best effort optimum routing and clustering organization solution depending on the current situation [4]. The feedback loop is closed to ensure that knowledge from the environment is retained 


\section{A. Basis of BDI-based Agent Infrastructures for SANETS}

The foundation of the experimental design is based on the Jadex, a Jade compliant agent development system designed by Universität Hamburg. Objected-oriented concepts form the basis of the BDI infrastructure, based on Java, a platform-agnostic programming language that allows implementation and execution on a Java-compatible runtime environment. The principles of BDI agencies is from the Foundation for Intelligent Physical Agents (FIPA), an organization created to standardize the development of artificially intelligent agents in software projects, of which Jadex is a category member [11].

The importance of the design of the middleware framework is from a utilitarian perspective; while the SANET agencies are designed in Jadex, the adherence to Jade compatibility standards ensures the framework can be easily ported to any Jade development environment [11]. As shown in Figure 1: Middleware Agencies in BDI Framework, the Multi-Agent System (MAS) middleware framework integrates the perceptions from the environmental space and transforms them into the agent space. The three core agencies in the framework consist of:

- Virtual agents are purely 'software' agents that emulates the behavior of a desired agent artifact for experimental practice, such as a sensor or actuator;

- Digital smart agents are embedded, self-contained agents that consist of both a microprocessor to execute agent tasks and is self-aware of its environment space;

- Analogous agents consist of analogue sensors attached to a microcontroller that is responsible for the agency and communication processes. It is designed to bind a compatibility space and allow legacy devices to interface with the environment.

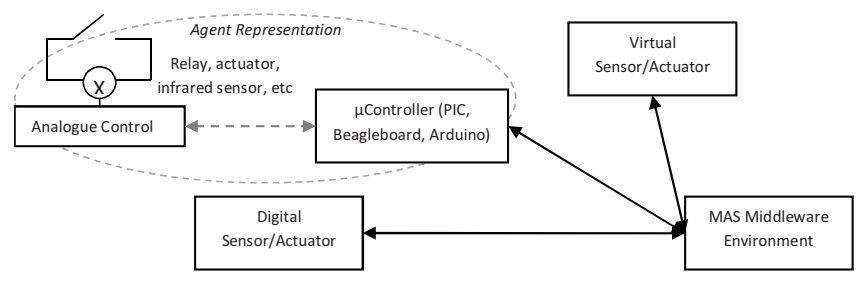

Figure 1: Middleware Agencies in BDI Framework

SANETs architectures for healthcare monitoring need to be service-oriented for composition, configuration and integration so the application can be designed efficiently from the data model, so the biomimetic model perspective can be projected onto a three dimensional space based on the POE Classification Model [1][2][20] as elaborated in Table 1. The POE model represents the different levels of organization, with POE standing for Phylogeny, Ontogeny and Epigenesis. In terms of the POE model, each software agent will encapsulate each of the different biomimetic models, in such a way that the functional scope of each agent is responsible for its own responsible parameters. For the same case in nature, the biological contexts for phylogeny, ontogeny and epigenesis are systemic processes that function independently from each other.
TABLE 1

POE MODEL AND SANET CORRELATIONS

Context and Correlation
Biological Context: Entail evolution of
species genetics.
SANET Correlation: This relates to the
implementation of heuristic problem solving
algorithms (Cooperative EKMs) and the
evolution of technology.
Biological Context: Concerned with cellular
growth process, multi-cellular organization,
cellular division and differentiation from the
parent to child cells. Each child cell
processes a copy of the original genome.
SANET Correlation: The perspective of the
domain space and calculation of EKM
activation energies.
Biological Context: This involves the
adaptation and learning processes. The
nervous, immune and endocrine systems are
characterized by epigenesis.
SANET Correlation: This space corresponds
to the facets or aspects of knowledge
acquisition by responding to stimuli and
weights adaption feedback.

\section{DOMAIN ANALYSIS OF SANETS}

\section{A. Cooperative EKMs with Agent-based Architectures}

Procedural controls are formed as a discrete set of commands to be used by reinforcement learning algorithms [18] to be selected from a library of heuristic functions for a distributed network in a healthcare concern. Reinforcement learning is driven by continuous control space functions [17], as indirect-mapping methods provide fluid decision choices than direct mapping. The accuracy in sensory stimuli control is important where external factors directly affect the network's robustness and reliability, allows for developing a feature map approach using co-operative EKMs with indirect mapping to improve the responsiveness of the tracking process [4][17].

By the direct-mapping technique, node inputs are mapped to sensory stimuli, with indirect-maps of sensory stimuli linked to a node with the utilization of control parameters, including energy discharge, signal strength and node roles as ordinary node, cluster head or location anchors. Meanwhile, by using the indirect-mapping technique, Continuous sensory stimuli space is mapped to the node; instead of direct-mapping that map continuous sensory stimuli space to the actual directives. Quality evaluators determine optimum clustering [5]. By using an indirectmapping process, a continuous processing space is established for each sensor and its corresponding sensory component, so that there is a direct correlation of data parameters for each sensor in the SANET space.

Event predication can be achieved using linear trajectory models in Euclidean space [14]. However, multidimensional problems outside of Euclidean space are sacrificed as a result, inclusive of network interference and ensuring energy efficiency. By not factoring the domain concern of including the problem set into a singular process model, the problem scope increases exponentially [1]. 
The solution is to transform the views and decompose them into a singular map of the highest activation energies or stimuli, which is achieved once the maximum value of the integration matrix is calculated for all EKMs for each of the input stimuli received from the SANET's environment. Insufficient sensory controls can result in unexpected or undesirable outcomes, leading to possible faults in navigating a route to the gateway [17][18]. When EKMs are established in the weighted-sum ensemble, a similar problem of unsolvable outcomes also takes place. This can be solved by using an indirect EKM mapping method, whereby the control vector is manipulated indirectly via a control parameter space [17].

The environmental concern shown in Figure 2: Cooperative EKM Process [4] for a given SANET domain can be summarized in the following statement tasks:

- For initial state described by input vector $u(0)$ in input space $U$, inclusive of the sensory perception;

- Adapt sequence of control vectors $\mathrm{c}(\mathrm{t}), \mathrm{t}=0$...t-1, in sensor control space $\mathrm{C}$ and solve activation energies;

- With resultant goal state elaborated by $\mathrm{u}(\mathrm{T}) \in \mathrm{U}$ that adapts the structure for a desired objective, thus reacting to the stimulus and changing the input state.

Using a multi-objective approach with co-operative EKMs as the modeling function has been adopted, with a reinforcement learning technique chosen on the basis of the situation or context [17] in an agent-based domain space. Interleaved EKMs that cooperate and compete to selforganize can enable a node to optimize in managing clustering and routing dynamically, whereby the node's output control is less than the total variable control available. Euclidean and weighted-sum ensemble methods have detrimental outcomes, even though a continuous sensory control space is implemented [14].

\section{B. Multi-Objective Meta-Heuristic Algorithm (MMA)}

The paradigm of meta-heuristics is taking the optimum yielded results of selected heuristic methods that are suitable for the problem domain in a healthcare environment. In particular, nature-inspired functions are most suitable when considering the learning capability of the healthcare network that is suitable for patient prediction models. Unlike with trajectory functions, which are concerned with local thresholds to yield a result, bio-inspired functions take a holistic view of the dynamic, evolving system. In essence, by combining a multi-dimensional approach to analyzing a given dataset, we can enhance the regenerative learning capability for any given heuristic optimization model. Current experiments for SANET modeling examine genetic algorithms and Particle Swarm Optimization (PSO) [8], as they both implicit-based reinforcement learning methods that attempt to seek a long term advantage through a representation set of scalar rewards. A filtration process is then applied to the mapping data to clear erroneous and randomized data introduced as a result of the algorithmic calculations, allowing for a clearer weighting adaption once a response is made to the stimuli.

The application of the heuristic algorithm follows the approach of applying EKM principles to the problem domain in SANETs [5][8]. This requires the modeling map to be translated into a training vector to be processed against a scalar rewards vector, which reflects the optimum energy or routing condition; depending on the current environmental concerns of the SANET network. The number of evolutions to determine an acceptable result will affect the quality of the process, but there requires a balance between obtaining a quality evaluation, which requires more processing time and is power intensive, with minimizing the time to process the heuristic function for resource conservation. The implementation method for metaheuristic functions in the experimental model is established procedurally in Table 2.

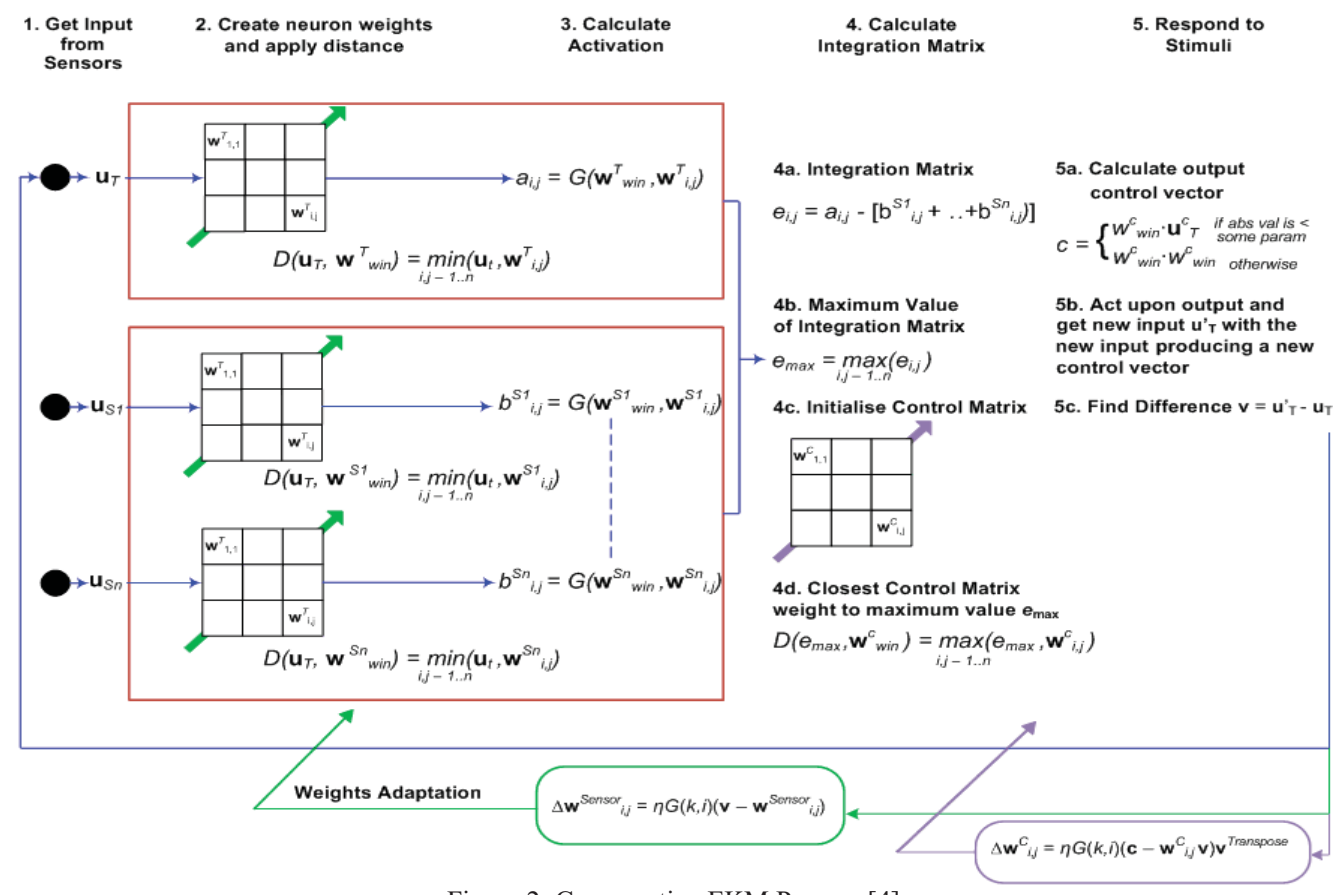

Figure 2: Co-operative EKM Process [4] 
TABLE 2

POE-STAged EXPERIMENTAL PROCESS

\begin{tabular}{|c|c|}
\hline POE Stage & Experimental Process \\
\hline $\begin{array}{c}\text { Stage 1: Model } \\
\text { Domain } \\
\text { Environment } \\
\text { (Phylogenical) }\end{array}$ & $\begin{array}{l}\text { - This stage is about performing an Unsupervised } \\
\text { Learning Heuristic on the environment. This } \\
\text { involves modeling the environmental concerns to } \\
\text { determine how the data should be organized for } \\
\text { optimum clustering and routing mechanisms. } \\
\text { The current experiment considers the use of EKMs } \\
\text { to model the SANET environment from the input } \\
\text { sensory perception. This autonomous process } \\
\text { occurs at the beginning. }\end{array}$ \\
\hline $\begin{array}{l}\text { Stage 2: Train } \\
\text { for }\end{array}$ & $\begin{array}{l}\text { This stage is about performing a Reinforcement } \\
\text { Learning Heuristic. This allows the environment }\end{array}$ \\
\hline $\begin{array}{l}\text { Environment } \\
\text { (Ontological) }\end{array}$ & $\begin{array}{l}\text { to be aware of its context, and learn from its own } \\
\text { experience and that of its cooperative actors. } \\
\text { The experiment incorporates genetic algorithmic } \\
\text { heuristics to achieve learning capability. Classical } \\
\text { techniques such as brute-force analysis is } \\
\text { computationally inefficient, and is infeasible to } \\
\text { consider all outcomes that lead to an optimum } \\
\text { solution, only the best-effort solution in time. }\end{array}$ \\
\hline $\begin{array}{c}\text { Stage } 3 \text { : Refine } \\
\text { Training Set } \\
\text { (Epigenetical) }\end{array}$ & $\begin{array}{l}\text { This stage is about factorizing the Training Set. } \\
\text { This optimizes the training set for a given learning } \\
\text { heuristic to a singular vector, reducing } \\
\text { invariability for long-term forecasting. } \\
\text { The current experiment considers generalized } \\
\text { optimization techniques to reduce noise and other } \\
\text { variability in the training set. This is necessary to } \\
\text { select an ideal candidate that establishes the fitness } \\
\text { condition for the SANET environment, such as } \\
\text { maintaining the minimum energy condition and } \\
\text { optimizing bandwidth. }\end{array}$ \\
\hline
\end{tabular}

\section{EXPERIMENTAL PROCEDURE}

The evaluation of co-operative EKMs on SANETs is executed using the framework conceived by Chaczko, et al [4]. The Jadex framework by Universität Hamburg, shown in Figure 3: Java Simulation Environment [5] allows for convenient monitoring and tracking of SANET events by pre-assigning event trajectories in the network field in an interactive manner.

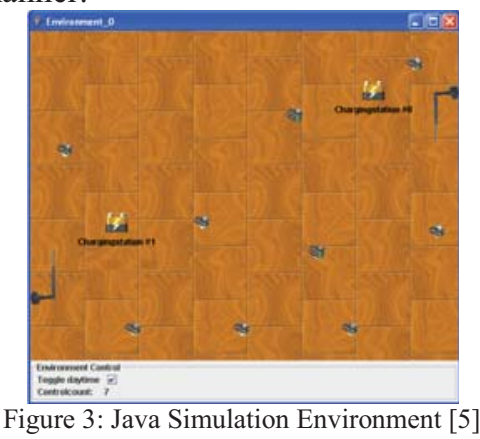

\section{A. General Assumptions}

- The topology of the SANET healthcare monitoring network is two-dimensional, such that altitude is constant and negligible between nodes.

- All nodes are powered at $100 \%$ capacity. Energy dissipation is calculated using inverse square law.

- The event trajectory is not predefined, such that the beginning and end points are calculated randomly using Fast Mersenne-Twister method.

- The network is considered to be interference free; as such RF communication concerns are not evaluated in the experimental model.

\section{B. Experimental Method}

The experiment is completed with the methodology by establishing the following simulation constraints:

1. A population of $n$ nodes is distributed randomly via Fast Mersenne-Twister, in a two-dimensional network of $100 \mathrm{~m}$ $\mathrm{x} 100 \mathrm{~m}$. Node populations tested include:
a. 100
d. 1000 ;
b. 250
e. 2000 ;
g. 4000
c. 500;
f. 3000 ;
h. 5000

2. An event trajectory is executed from a point in the network area; of which the test path course is either:

a. Linear Path: A linear path consists of an event trajectory where the entry and exit point from the area is of constant gradient.

b. Arc-formation Path: Arc-formation consists of an event trajectory where the entry and exit point will either be increasing or decreasing in gradient, forming a circle segment.

c. Pseudo-random Path: A pseudo-random path using the Mersenne-Twister method combines 2(a) and 2(b) at various points throughout the trajectory, until it reaches the exit point.

3. The algorithm selects the route from the node in range of the approximate trajectory to be established to the sink; such that the closer the algorithm is to calculating the event path, the more optimum the route will be to establish successful negotiations.

a. Co-operative EKMs with PSO: Co-operative EKMs use an indirect-mapping SOM map to train the control parameters in which to converge at the final trajectory point; in such a fashion to actively train the neural network to seek positive outcomes to determine a route from the trajectory's path to the sink.

b. Co-operative EKMs with Agent-based Architectures: In conjunction with Co-operative EKMs, a filtrating mechanism is applied to the weights adaptation map to assist in the event tracking functions.

In conjunction, the reinforcement learning heuristic applied to the SOM map as a training vector set, and a vector test set is applied to maintain accuracy of path estimation. Using Particle Swarm Optimization [8], the shortest Euclidean distance between the specific trajectory point and the nearest neighboring node is nominated within the routable path to the sink or health repository.

4. The experiment is executed for 1000 iterations to calculate the mean rate of successful identification of the trajectory's target point, when the simulated event exits the area:

a. A maximum margin of error is a $2 \mathrm{~m} \times 2 \mathrm{~m}$ area where final approximate point is found.

b. A successful identification is where the final endpoint is within a $95 \%$ confidence interval of the entire network. Any estimation outside of this threshold is identified as a failed identification.

\section{EXPERIMENTAL RESULTS}

The results demonstrate that in comparison to cooperative EKMs with PSO utilizing agent-based architectures, the final results are positive when pseudorandom trajectory tracking is required. While standard Cooperative EKMs perform adequately in the given scenarios; co-operative EKMs demonstrates an improvement in the identification rate over standard co-operative EKM algorithms. This is evident with a greater node population, as the granularity of determining a nearest neighboring node to route is reduced for the fixed size of the network. 
As shown in Figure 4: Experimental Results of Detection there is a $35 \%$ relative improvement in the average mean detection rates with agent-based optimization for the pseudo-random trajectory compared to standard cooperative EKMs. As co-operative EKM demonstrate improvement over passive learning techniques, current prediction rates are sufficient for routing estimation capability, which indicate the process of refining the training set for remodeling is necessary to improve the SANET routing condition.

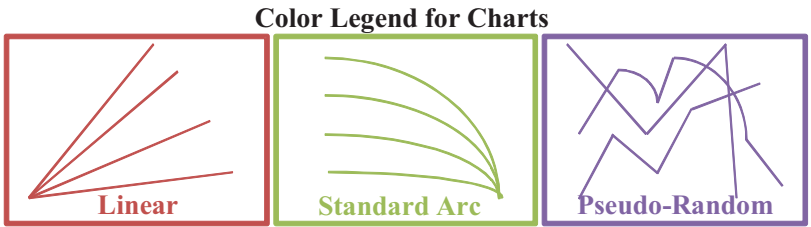

Co-operative EKMs
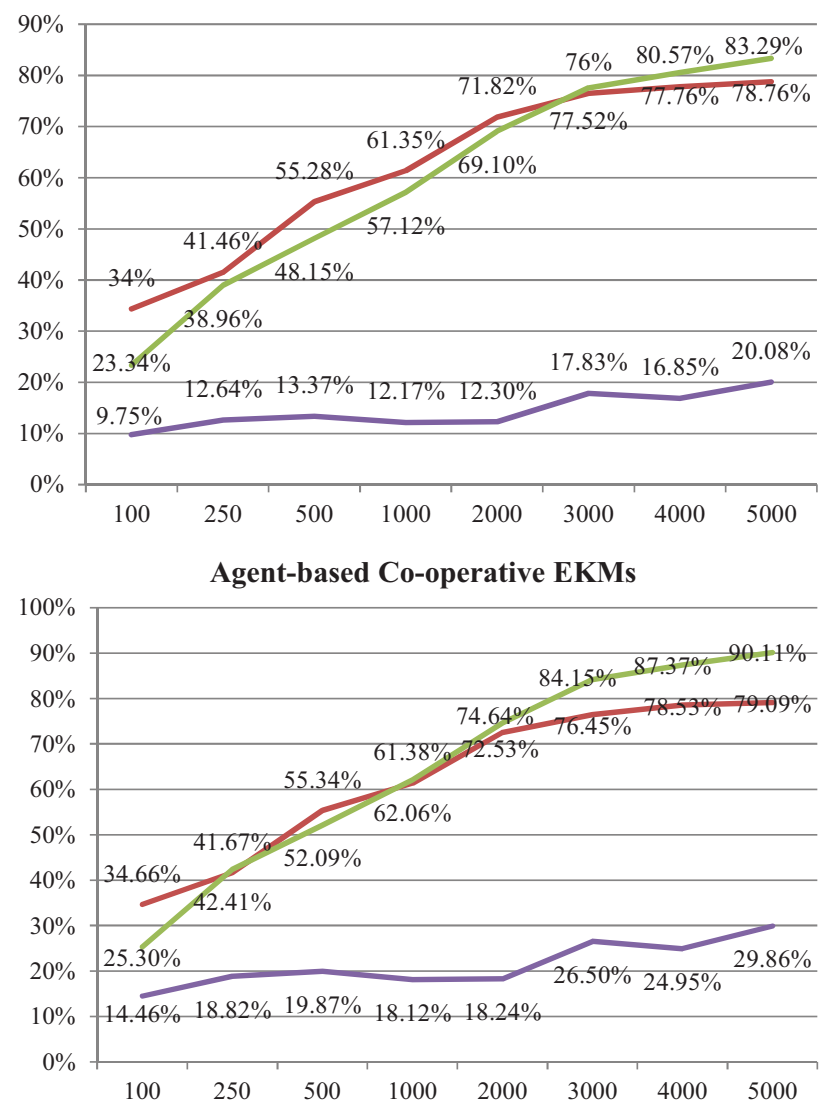

Figure 4: Experimental Results of Detection Success

The analysis of co-operative EKMs as shown below in Table 3 and 4, when assessed in terms of performance of pseudo-random tracking, requires more analysis into the algorithmic procedure. In particular, the thresholds established for determining positive or negative learning reinforcement is an issue that needs to be evaluated for an in-depth assessment. The tolerance levels used to calculate the thresholds is important, as subtle variations in tolerance may yield undesirable results. As a case in point, reducing tolerance levels too far will result in the inflexibility of the algorithm to adapt to changes the event trajectory; the corollary is that generous tolerance levels will yield undesirable tracking results when noise or faulty nodes produce invalid sensory data.
Furthermore, the use of Clifford algebraic forms can be used in a SANET system in terms of extending the geometric form of the network's geometric structure [14][15]. The quaternions of the network can be extended for $4^{\text {th }}$ dimensional rotation groups, which serves as an ideal base for energy conservation and core attributes of a SANET network. Although the current simulation framework does not incorporate Clifford algebraic forms as an optimization technique, the modular design of the simulation environment will enable incorporation of this technique in the next iteration of the healthcare framework.

TABLE 3 AND 4

COMPARATIVE ASSESSMENT AND EVALUATION

\section{CO-OPERATIVE EKMS WITH PSO}

\begin{tabular}{|c|c|}
\hline Assessment & Evaluation \\
\hline $\begin{array}{l}\text { Quantitative } \\
\text { Assessment }\end{array}$ & $\begin{array}{l}\text { Demonstrates an indirect-mapping EKM } \\
\text { can provide detection to optimize for local } \\
\text { obstruction and global target identification } \\
\text { concerns in a distributed sensor space. } \\
\text { Results show a smoother tracking } \\
\text { mechanism to monitor events in real-time, } \\
\text { compensating for random events [17][19]. } \\
\text { Co-operative EKMS with PSO, in } \\
\text { conjunction with an enhanced training set } \\
\text { refinement mechanism, yields more } \\
\text { consistent results as due to an optimal } \\
\text { training vector. }\end{array}$ \\
\hline \multicolumn{2}{|c|}{ CO-OPERATIVE EKMS WITH AGENT-BASED ARCHITECTURES } \\
\hline Assessment & Evaluation \\
\hline $\begin{array}{l}\text { Qualitative } \\
\text { Assessment }\end{array}$ & 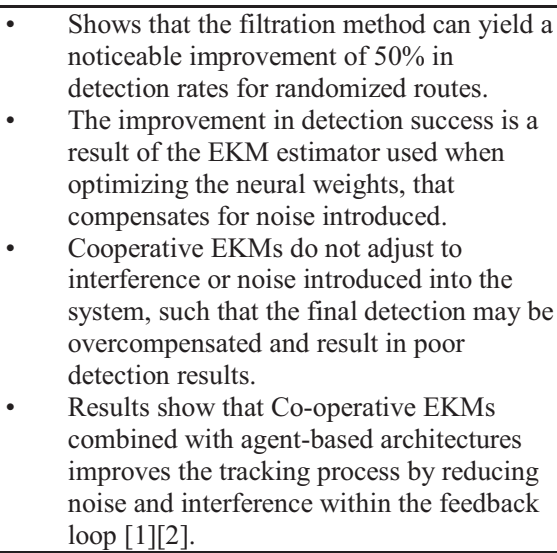 \\
\hline
\end{tabular}

As a consequence, the potential of Co-operative EKMs to identify events within a SANET network is evident; but as with all passive learning heuristic methods, a heuristic ensemble approach using MMA is necessary to train the algorithm to evaluate and determine the tolerance thresholds that are most suitable for the current conditions. The implementation of co-operative EKMs with alternative heuristic algorithms such as genetic algorithms will need to be considered in future to evaluate improvement in the mean identification rate. Furthermore, a feedback mechanism incorporating training set factorization is required to minimize error rates and ensure a consistent set of data outcomes, and allow for more fluid responses in the routing and tracking network functions. 


\section{RESULt ANALYSIS}

\section{A. Cooperative EKMs with Agent-based Architectures}

The notable variability in the identification rate indicates a need to improve the quality of training mechanisms to reinforce positive selection processes, so the aggregation of the final routing selection is optimal for the system environment. Furthermore, the implementation of refining the training set is necessary to ensure that the SANET modeling functions do not introduce internally generated erroneous data that will impact on the system's ability to make future predictions based on historical trends. The relative improvement of $50 \%$ on traditional cooperative EKMs is significant to note; with the aim being a yield in the trajectory projection to ensure results are within the $95 \%$ confidence interval in tracking projections. In particular, further examination is required to determine the trade-off between yielding an efficient route which will be more computationally intensive, or a best-effort route that will be more energy conservative. However, the distributed nature of SANETs means that computational calculations can take place for each routing hop, so that while the local calculations are considered for each route, a global picture is established for the entire route to the gateway or central healthcare repository.

\section{CONCLUSION}

The method of adaptive SANET management for healthcare monitoring with co-operative EKMs coupled with agent-based architectures is beneficial, with the current results demonstrating indirect-mapping EKM generating more proficient routing and clustering conditions when compared to direct-mapping EKMs. Furthermore, the control parameters of the indirect-mapping EKM can be enhanced with Rao-Blackwell algorithms to allow convergence and better optimization, with choices in the reinforcement learning technique providing context-aware outcomes to improve the reinforcement learning capability for patient monitoring.

\section{REFERENCES}

[1] Chaczko, Z. et al, (2005), A Bio-inspired Telecollaboration Service Taxonomy: Usability Related Concerns, Broadcom 2008

[2] Chaczko, Z. et al, (2007), NICE Models of Biomimetic Software Systems, CSIRO ICT Centre Conference.

[3] Chaczko, Z., Davis, D., Mahadevan, V. (2004), New Perspectives on Teaching and Learning Software Systems Development in Large Groups, IT Higher Education \& Training 2004, Istanbul, Turkey

[4] Chaczko, Z; Chiu, C. (2009), Cooperative EKMs for Wireless Sensor Networks, Eurocast 2009, Vol.12, pp. 304-305, published by Gran Canarias Las Palmas University Press

[5] Chaczko, Z; Nikodem, J; Klempous, R; Nikodem, M; Sensors Localisation Methods in the COSA Framework (2007), AusWireless: The 2nd International Conference on Wireless Broadband and Ultra Wideband Communications, pp. 60

[6] Chiu C.C., Chaczko, Z., Moses, P. (2009), Advanced Extended Kohonen Mapping Modelling Techniques for Sensor Actor Networks, 4th International Conference on Broadband Communication, Information Technology \& Biomedical Applications (BroadBandCom 2009), Wroclaw, Poland, 15-18 July 2009

[7] Chiu C.C., Chaczko, Z., Moses, P. (2009), Sensor Actor Network Modeling utilizing the Holonic Architectural Framework, Electronics \& Telecommunications Quarterly, International Journal of Electronics $\&$ Telecommunications (KEiT-PAN 2009), Volume 55, Issue 4
[8] Clerc, Maurice (2006), Particle Swarm Optimization: ISTE (International Scientific and Technical Encyclopedia), translated from L'optimisation par essaims particulaires. Versions paramétriques et adaptatives, Hermès Science, 2005.

[9] Ghosh, J. and Nag, A. (2001). An overview of radial basis function networks. In R. J. Howlett and L. C. Jain, editors, Radial Basis Function Networks: New Advances in Design, pp. 1-36. PhysicaVerlag, New York

[10] Health Level Seven (HL7) Inc, http://www.hl7.org/, Last Visited $22^{\text {nd }}$ January 2010

[11] Jadex Development Framework, Last Visited $11^{\text {th }}$ June 2011, http://vsis-www.informatik.uni-hamburg.de/projects/jadex/

[12] Kaelbling, L. P., Littman, M. L., and Moore, A. W. (1996). Reinforcement learning: A survey. J. Artificial Intelligence Res., pp. 237-285

[13] Kohonen, T. (2000). Self-Organizing Maps. Springer, New York, 3rd Edition.

[14] Lawson, H. B., Michelsohn, M.L. (1989). Spin Geometry, Princeton, NJ: Princeton University Press

[15] Lounesto, P. (2001), Clifford algebras and spinors, Cambridge: Cambridge University Press

[16] Low, K. H., Leow, W. K., and Ang, Jr., M. H. (2004). Task allocation via self-organizing swarm coalitions in distributed mobile sensor network. In Proc. 19th National Conference on Artificial Intelligence (AAAI-04), pp. 28-33

[17] Low, K. H., Leow, W. K., and Ang, Jr., M. H. (2005), An Ensemble of Cooperative Extended Kohonen Maps for Complex Tasks In Neural Computation, Vol. 17, Issue 6, pp. 1411-1445, published by MIT Press

[18] Mill'an, J. del R., Posenato, D., and Dedieu, E. (2002). Continuousaction Qlearning. Machine Learning, pp. 249-265.

[19] Sharkey, A. J. C. and Sharkey, N. E. (1997). Combining diverse neural nets. Knowledge Engineering Review, pp. 231-247.

[20] Sipper, M. et al, (1997), A Phylogenetic, Ontogenetic, and Epigenetic View of Bio-Inspired Hardware Systems, IEEE Transactions on Evolutionary Computation 1-1, pp. 83-97

[21] Szyperski, C. (1998), Emerging component software technologies - a strategic comparison, Software - Concepts and Tools, Vol 19, No 1, p2-10. 